\title{
Oxidative damage and DNA damage in lungs of an ovalbumin- induced asthmatic murine model
}

\author{
Yuanfang Wang ${ }^{1,2}$, Jiangtao Lin ${ }^{1,2}$, Jun Shu ${ }^{3}$, Hong Li $^{3}$, Zhencui Ren ${ }^{2}$ \\ ${ }^{1}$ Peking University China-Japan Friendship School of Clinical Medicine, Beijing 100029, China; ${ }^{2}$ Department of Respiratory Diseases, ${ }^{3}$ Institute of \\ Clinical Medical Science, China-Japan Friendship Hospital, Beijing 100029, China \\ Contributions: (I) Conception and design: Y Wang, J Lin; (II) Administrative support: J Lin; (III) Provision of study materials or patients: Y Wang, J \\ Lin, J Shu, H Li; (IV) Collection and assembly of data: Y Wang, J Lin, Z Ren; (V) Data analysis and interpretation: Y Wang, J Lin; (VI) Manuscript \\ writing: All authors; (VII) Final approval of manuscript: All authors. \\ Correspondence to: Jiangtao Lin. Peking University China-Japan Friendship School of Clinical Medicine, Beijing 100029, China. \\ Email: jiangtao_1@263.net.
}

Background: Asthma is characterized to chronic airway inflammation. However, the role of oxidative damage and DNA damage in the pathophysiology of asthma have rarely been studied. On the other hand, there are evidences that DNA-dependent protein kinase (DNA-PK) participates in DNA damage repair and regulates innate immune responses and proinflammatory signaling pathways.

Methods: After ovalbumin (OVA)-induced asthmatic murine model was established, airway hyperresponsiveness (AHR), total and differential bronchoalveolar lavage fluid (BALF) cell counts. IL-4, IL-8, IL-13 and TNF- $\alpha$ were chosen to evaluate the airway inflammation, and oxidative damage indicators levels (8-isoprostane and 8-OhdG) in BALF were measured. Alkaline comet assay was conducted to detected DNA damage. Histological analysis was conducted after hematoxylin and eosin (HE) straining, and proteins were extracted for 3-nitrotyrosine (3-NT) detection and immunoblotting.

Results: AHR, infiltration of inflammatory cells and pro-inflammatory cytokine levels in lungs were significantly higher in asthmatic mice. OVA challenge resulted in robust increase in 3-NT, 8-isoprostane and $8 \mathrm{OHdG}$ in lungs, which represented oxidative damage level. DNA damage and repair proteins levels in asthma were also increased. NU7441 aggravated the DNA damage level. However, it suppressed infiltration of lung inflammatory cells and inflammatory cytokine levels, suggesting that DNA-PK may be a potential target for treatment of allergic asthma.

Conclusions: Our study showed that oxidative damage and DNA damage existed in the airway of asthmatic mice. NU7441 augmented DNA damage level, and moreover, it also attenuated infiltration of inflammatory cells and pro-inflammatory cytokine levels in asthmatic lungs.

Keywords: Asthma; oxidative damage; DNA damage; airway inflammation

Submitted Mar 21, 2018. Accepted for publication Jul 09, 2018.

doi: $10.21037 /$ jtd.2018.07.74

View this article at: http://dx.doi.org/10.21037/jtd.2018.07.74

\section{Introduction}

Asthma is one of the most common respiratory diseases in the world, which can affect human health seriously. There are about 300 million asthma patients in the world (1). Moreover, According to the World Health Organization, there will be 400 million asthma patients in the world in 2025 , which will bring a huge burden to society (1).
Despite its prevalence, there is little research on the cytotoxicity of asthma in pathophysiology of asthma. The main features of asthma are reversible bronchial obstruction and airway hyper-responsiveness (AHR) caused by chronic airway inflammation (2). Reactive oxygen species and nitrogen species (RONS), which are produced by inflammatory cells in the asthmatic airway, may cause oxidative damage 
to lipids, proteins, and nucleic acids and exacerbate asthma progression (3). Here we chose 8-isoprostane, $8-\mathrm{OHdG}$ and $3-\mathrm{NT}$ as indicators to measure oxidative damage in lipids, nucleic acids, and proteins respectively.

Moreover, the airway inflammation and oxidative damage are very likely to result in a genetic toxicity to the airway epithelial cells. Previous studies have found that base lesions and DNA single-strand breaks (SSBs), which are induced by nitric oxide and peroxynitrite are the major mechanisms of large-scale gene sequence rearrangement $(4,5)$. DNA double-strand breaks (DSBs) are one of the most serious genetic toxicity forms of damage. Fast and accurate repair of DSBs plays a key role in genome stability (6). Homologous recombination (HR) and nonhomologous end joining (NHEJ) are the two major repair pathways of DNA DSBs. DNA-PK is a key enzyme involved in NHEJ, which is composed of a $450-\mathrm{kDa}$ catalytic subunit (DNA-PKcs) and two DNA-binding subunits (Ku70 and Ku80). We used NU7441, a well-characterized inhibitor of DNA-PKcs, to further explore the role of DNA damage repair in the pathogenesis of asthma.

There are plenty of DNA-PKcs in human body. But only less than $1 \%$ of them are needed in DNA damage response (7), suggesting that DNA-PK also participates in other processes in addition to DSB repair. Some studies concluded that DNA-PK had the ability to regulate innate immune responses and pro-inflammatory signaling pathways $(8,9)$. After that, researchers also found that DNAPK affected the drug resistance of glioblastoma through the PI3K-Akt pathway and drug insensitivity can be reversed by NU7441 (10). These results mentioned above have shown that DNAPK is a potential new target for asthma treatment. In this study, we establish an asthmatic murine model challenged by OVA to study the oxidative damage and DNA damage in asthmatic mice as well as the role of DNAPKcs inhibitor NU7441 in it. We found that asthma led to a significant increase in oxidative damage and DNA damage in lung tissues. NU7441 worsened the DNA damage in asthmatic mice as an inhibitor of DNA repair, but it surprisingly suppressed OVA-induced airway inflammation, suggesting NU7441 as a novel treatment research area for asthmatic patients.

\section{Methods}

\section{Animals}

Sixty specific pathogen free (SPF) female BALB/c mice,
6 weeks old and $(18 \pm 2) \mathrm{g}$ weight, purchased from Beijing HFK Bio-technology Co. Ltd. were divided into 4 groups equally and randomly: normal group, asthmatic group (OVA group), asthmatic mice treated with NU7441 (NU group), and asthmatic mice treated with vehicle for NU7441 (OVA + vehicle group). All mice were raised in a SPF residence, which satisfied the People's Republic of China National Standard (GB 14925-2010, China). This study was approved by the Animal Ethics Committee of China-Japan Friendship Hospital (ID of the ethic approval: 170105).

\section{Experimental protocol}

All 4 groups except normal group were sensitized with $100 \mu \mathrm{L}$ sensitization liquid [20 $\mu \mathrm{g}$ ovalbumin OVA (Grade VI, Sigma, USA) and $3 \mathrm{mg} \mathrm{Al}(\mathrm{OH})_{3}$ emulsified in saline] per mice by intraperitoneally on days $0,7,14$, and challenged with $1 \%$ OVA aerosol for $30 \mathrm{~min}$ from days 21 . The normal group was sensitized and challenged with saline instead of OVA. NU7441 (Santa Cruz Biotechnology, Santa Cruz, CA) at dose of $10 \mathrm{mg} / \mathrm{kg}$ or vehicle [5\% dimethyl sulfoxide (DMSO)] was administered intraperitoneally once a day on days 21 to days 30, $30 \mathrm{~min}$ after OVA challenge. The dosage and route of NU7441 administration were determined based on previous study (11), since the plasma pharmacokinetics of the drug was examined. The experimental protocol diagram was shown in Figure 1.

\section{Measurement of AHR}

The invasive AHR measurement was performed $24 \mathrm{~h}$ after the last challenge as previously described (12), and 6 mice per group were anesthetized with $1 \%$ pentobarbital sodium (Merck, Germany) before the performing. AHR to methacholine (Sigma, USA) was measured by the AniRes 2005 lung function system (Bestlab, AniRes 2005, Version 3.0, China). Lung function was recorded as the level of inspiratory resistance (RI), expiratory resistance (RE) and dynamic compliance (Cdyn).

\section{BALF cell counting and classification}

The left 9 mice were anesthetized to get prepared for tracheal intubation and blood collection from heart. The blood samples were stored at $-80{ }^{\circ} \mathrm{C}$ for subsequent analyses. After that, the right lung was instilled 5 times with $1 \mathrm{~mL}$ pre-cooled $\left(4^{\circ} \mathrm{C}\right)$ saline to collect BALF $(0.6-0.7 \mathrm{~mL})$. 


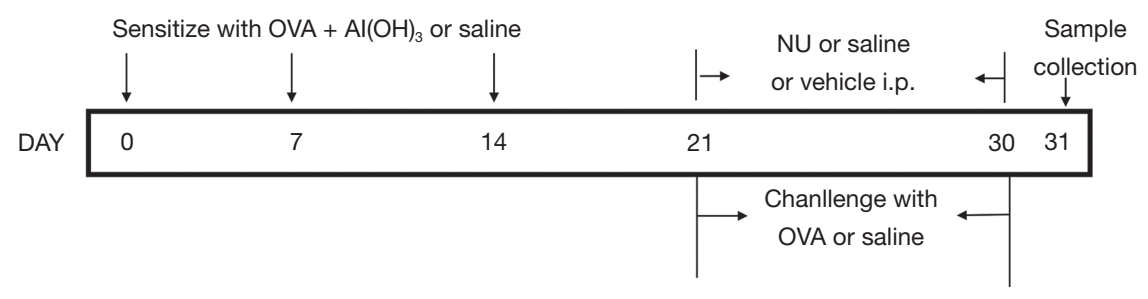

Figure 1 Experimental protocol: Mice were sensitized with OVA or saline on days 0, 7, 14. From days 21, mice were challenged with $1 \%$ OVA aerosol or saline for 10 days. NU7441 or vehicle or saline was administered on days 21 to days 30. Twenty-four hours later, mice were anesthetized for sample collection. OVA, ovalbumin.

Then, BALF was centrifuged at 2,000 rpm for $7 \mathrm{~min}$ to separate supernatant, which was collected and stored at $-80{ }^{\circ} \mathrm{C}$ for subsequent analyses. The precipitates were resuspended in $0.1 \mathrm{~mL}$ phosphate buffered saline (PBS, $0.02 \mathrm{~mol} / \mathrm{L}, \mathrm{pH}$ 7.2) for cell counting and classification. Total cell number was counting on a Fuchs-Rosenthal Chamber. Cell classification was performed after WrightGiemsa staining (Baso Diagnostics Inc., China).

\section{Measurement of cytokines, 8-isoprostane and 8-OHdG in $B A L F$}

Cytokines (IL-4, IL-13, IL-8, and TNF- $\alpha$ ), 8-isoprostane and 8-OHdG levels were detected in BALF with enzyme linked immunosorbent assay (ELISA) kits (R \& D Systems, Minneapolis, MN, USA).

\section{Histopathological analysis}

The left lungs were infiltrated in 10\% formalin immediately for 72 hours after the BALF. After that, they were embedded in paraffin and 5- $\mu$-thick sections were sliced. Histopathological changes were observed after HE staining using a brightfield microscopy (ZEISS Axioplan 2 imaging MOT, Jena, Germany) by two pathologists.

\section{Immunobistochemistry assay}

The paraffin sections were also used to test the levels of $\gamma \mathrm{H} 2 \mathrm{AX}$ (Abcam, Cambridge, UK) by immunohistochemistry assay. Pre-experiment was conducted to optimize the concentration of $\gamma \mathrm{H} 2 \mathrm{AX}$ antibody. If a cell or tissue was turned into yellow or brown, it would be recorded as positive immunostaining. Histological assay was analyzed by Image Pro Plus6.0 (Media Cybernetics, Rockville, MD, USA) at 400× magnification. Measurements were performed blindly and under similar light, gain, offset, and magnification conditions.

\section{3-nitrotyrosine (3-NT) in lung tissues}

The remaining lungs were homogenized with a glass homogenizer before cutting into small pieces. And an ultrasonication (Scientz, Ningbo, China) was also used to rupture the cell membranes. Then the supernatant was taken after centrifuging for $15 \mathrm{~min}$ at 5,000 rpm for analysis. Lung levels of 3-NT were measured with a ELISA kit (Blue Gene, Shanghai, China) according to the protocol instructions.

\section{Immunoblotting}

The right lung tissues were stored at $-80{ }^{\circ} \mathrm{C}$ for immunoblotting as described after being cleaning up connective tissues and lymph nodes (13). Antibodies used were targeted at Ku70, Rad51, PARP-1 (Santa Cruz Biotechnology, Dallas, Tex, USA), $\gamma \mathrm{H} 2 \mathrm{AX}$ (Abcam, Cambridge, UK), and $\beta$-actin (Abcam, Cambridge, UK).

\section{Comet assay}

The comet assay was performed essentially as described by Hininger (14). After quick thawing in a water bath at $37^{\circ} \mathrm{C}$, the frozen total blood samples were mixed with $\mathrm{Ca}^{2+}$ and $\mathrm{Mg}^{2+}$ free PBS at a ratio of $1: 1$ up to $100 \mu \mathrm{L}$. Then the mixer was embedded in a layer of $100 \mu \mathrm{L} 0.75 \%$ low melting point agarose gel on frosted slides. After that, it was immersed in a cell lysis buffer $(2.5 \mathrm{~mol} / \mathrm{L} \mathrm{NaCl}$, $0.1 \mathrm{~mol} / \mathrm{L}$ Na2EDTA, $10 \mathrm{mmol} / \mathrm{L}$ Tris-Hcl, $\mathrm{pH}$ 10.0-10.5, with freshly added $1 \%$ Triton X-100 and $10 \%$ DMSO) for 1 hour in dark. Subsequently, the slides were incubated in freshly-made alkaline buffer $(300 \mathrm{mmol} / \mathrm{L} \mathrm{NaOH}$ and $1 \mathrm{mM}$ EDTA, pH 12.6) for 30 minus to unwind the DNA. After that, the samples were electrophoresed for $25 \mathrm{~min}$ at 25 


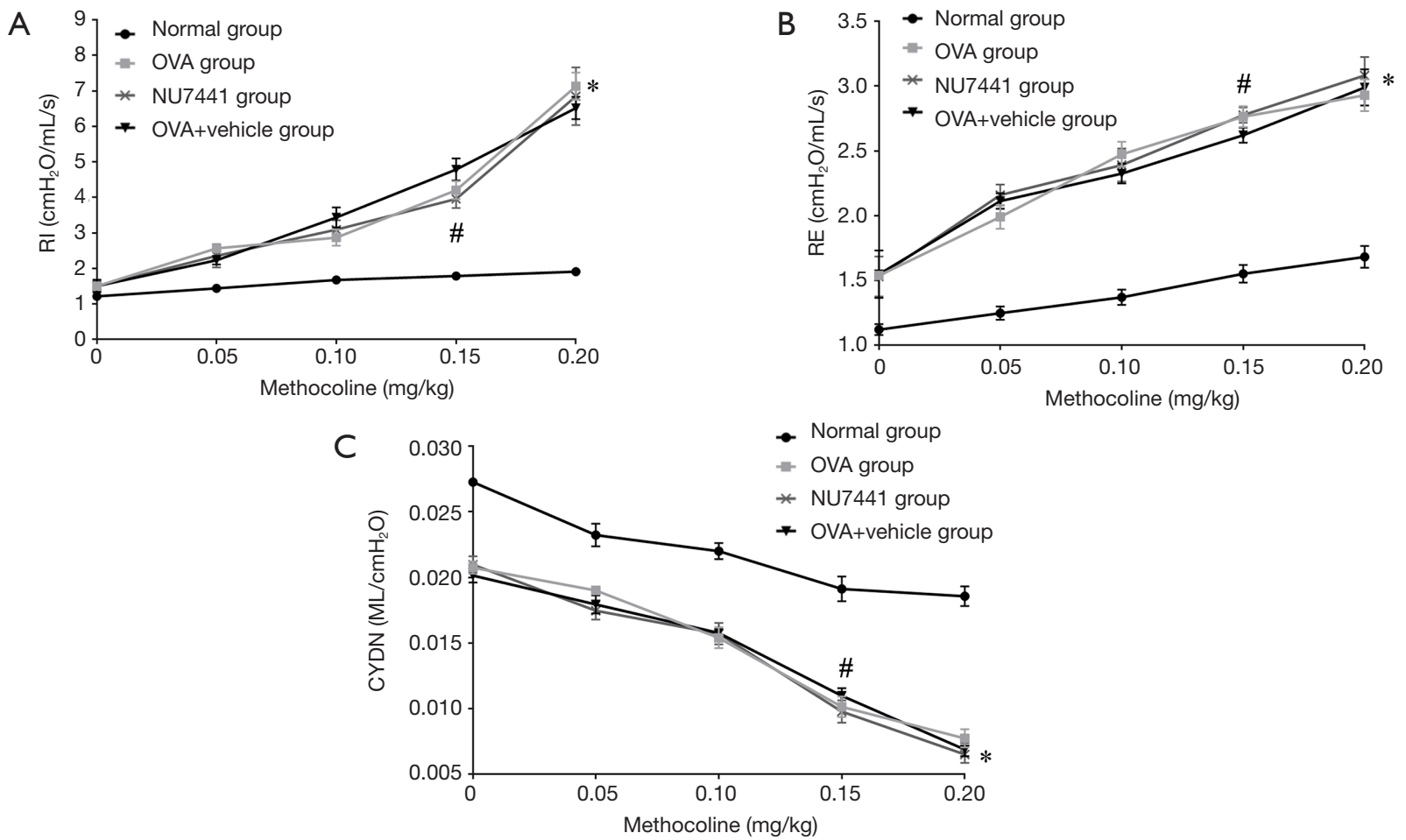

Figure 2 The invasive AHR measurement was performed $24 \mathrm{~h}$ after the last challenge (n=6 per group). RI (A), RE (B) and Cdyn (C) were used to assess the AHR. All values are shown as means \pm SEM. *, OVA group vs. Normal group, $\mathrm{P}<0.001$; \#, NU7441 group vs. OVA + vehicle group, $\mathrm{P}<0.05$. OVA, ovalbumin; AHR, airway hyper-responsiveness; RI, inspiratory resistance; RE, expiratory resistance; Cdyn, dynamic compliance; SEM, standard error of the mean.

volts $(0.90 \mathrm{~V} / \mathrm{cm})$ and $300 \mathrm{~mA}$. Then the slides were immersed in PBS for 15 min twice to neutralize alkali. Finally, the slides were stained with $20 \mu \mathrm{L}$ ethidium bromide solution $(2 \mu \mathrm{g} / \mathrm{mL})$. All the steps were performed in a dark environment.

\section{Statistical analysis}

Data are expressed as means \pm standard error of the mean (SEM). One-way ANOVA Dunnett test was chosen for statistical analysis between groups. $\mathrm{P}$ values $<0.05$ were considered to be statistically significant. All data were analyzed using the SPSS 19.0.

\section{Results}

\section{Assessment of airway responsiveness}

As shown in Figure 2, OVA-challenged Mice had higher $\mathrm{RI}$ and RE and lower Cydn than the Normal group (OVA, OVA + vehicle, NU7441 vs. Normal, $\mathrm{P}<0.001)$. Compared with OVA group, RI, RE, and Cdyn were only significantly changed at the concentration of $0.15 \mathrm{mg} / \mathrm{kg}$ in NU7441 mice $(\mathrm{P}<0.05)$. There was no significant difference in those data between OVA group and Vehicle group.

\section{The number of total and differential cell counts in the BALF}

The number of total and differential number of cells in OVA and OVA + vehicle group was the highest, followed by NU7441 group, and was the lowest in Normal group (Figure 3). Compared with Normal group, the number of eosinophils in OVA group was much higher $(\mathrm{P}<0.05)$. NU7441 suppressed both the neutrophil and eosinophil counts compared with OVA group $(\mathrm{P}<0.05)$. Different cell types stained with Wright-Giemsa were shown in Figure 4.

\section{Histopathological features of lung tissues}

Histopathological changes were showed in Figure 5. There 
were obvious features of airway inflammation in groups which were exposed to OVA, including inflammatory cell infiltration in the vicinity of bronchioles, alveolar cavity,

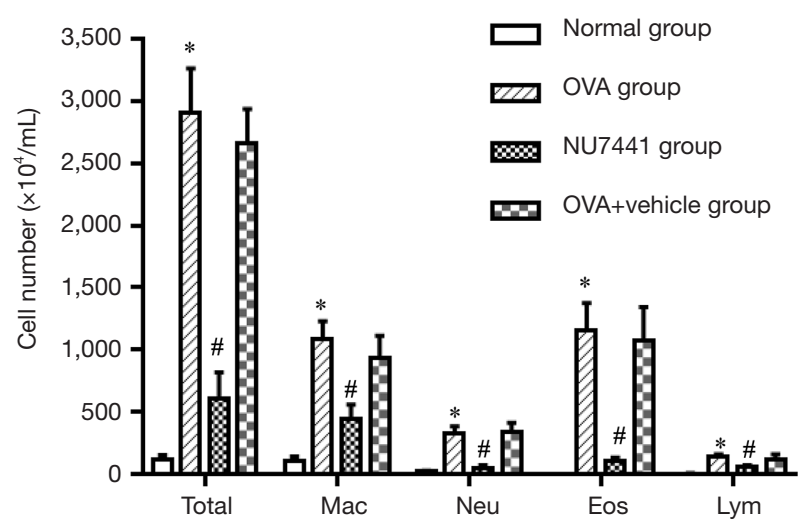

Figure 3 Total cell number of the BALF was counting on a FuchsRosenthal Counting Chamber after trypan blue staining ( $\mathrm{n}=9$ per group). Numbers of neutrophils (Neu), eosinophils (Eos), macrophages (Mac), and lymphocytes (Lym) were calculated after Giemsa staining. *, OVA group vs. Normal group, $\mathrm{P}<0.001$; \#, NU7441 group vs. OVA + vehicle group, $\mathrm{P}<0.001$. OVA, ovalbumin; BALF, bronchoalveolar lavage fluid. alveolar septum and surrounding vessels, irregular enlarging of the residual alveolar cavity, and mucus impacted in alveolar cavity. There was no pathological change observed in the Normal group. NU7441 attenuate the inflammatory cell infiltration and cilia damage significantly.

\section{$I L-4, I L-8, I L-13$, and TNF- a levels in BALF}

As seen in Figure 6, cytokines in OVA group were much higher than Normal group $(\mathrm{P}<0.05)$, which validated the murine asthma model was successful established. Compared with OVA group, NU7441 markedly reduced IL-4, IL-8, IL-13, and TNF- $\alpha$ levels $(\mathrm{P}<0.05)$, exhibiting a strong antiinflammatory effect.

\section{Oxidative damage in airway}

8-isoprostane and 8-OHdG in BALF and 3-NT in lung tissue were detected to measure oxidative damage in airway (Figure 7). OVA exposure caused a significant increase in these 3 indicators, which represented oxidative damage to lipids, nucleic acids and proteins $(\mathrm{P}<0.05)$.

However, NU7441 didn't augment this oxidative damage.
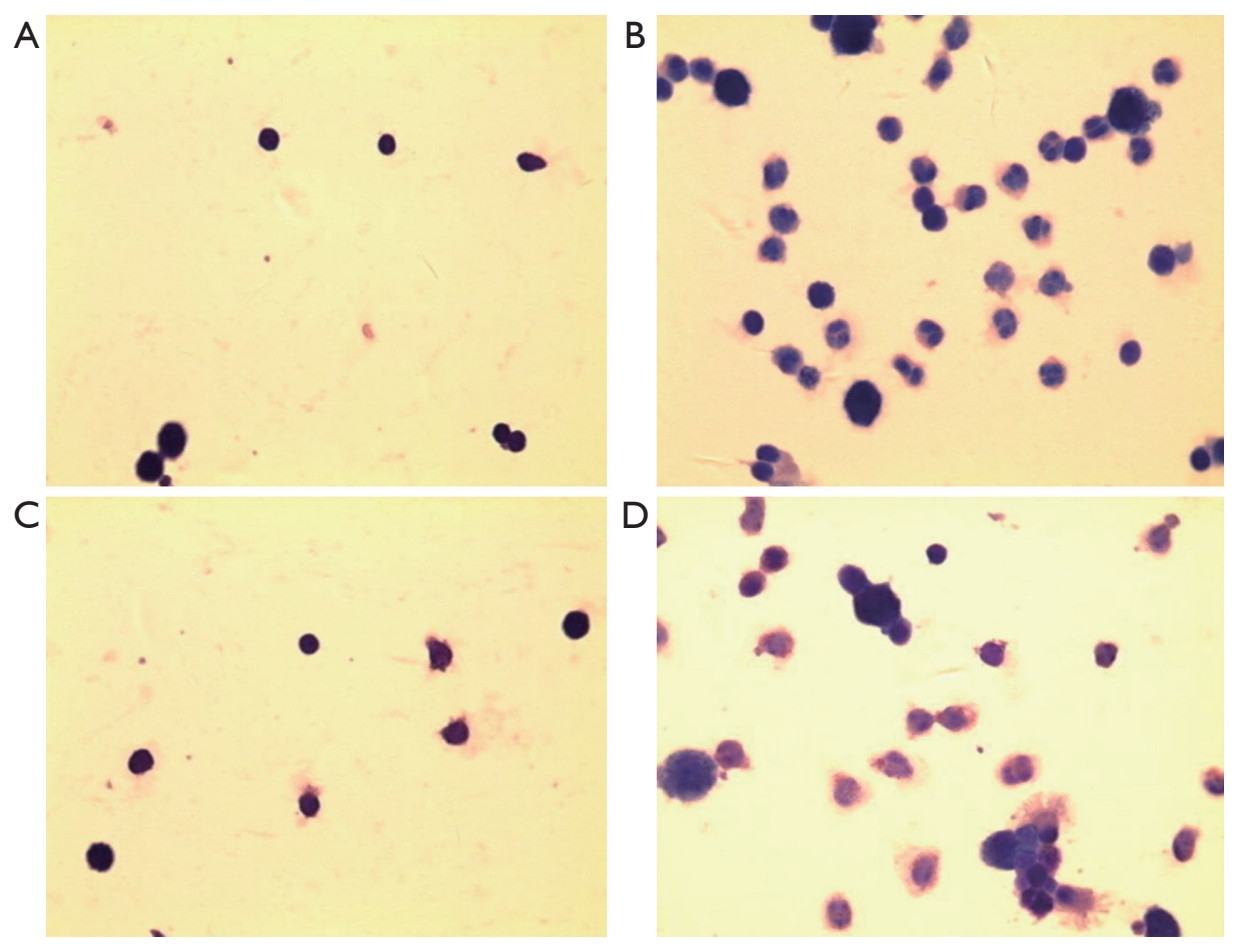

Figure 4 The images of BALF cytospin at 1,000× magnification. (A) Normal group; (B) OVA group; (C) NU7441 group and (D) OVA + vehicle group. BALF, bronchoalveolar lavage fluid; OVA, ovalbumin. 

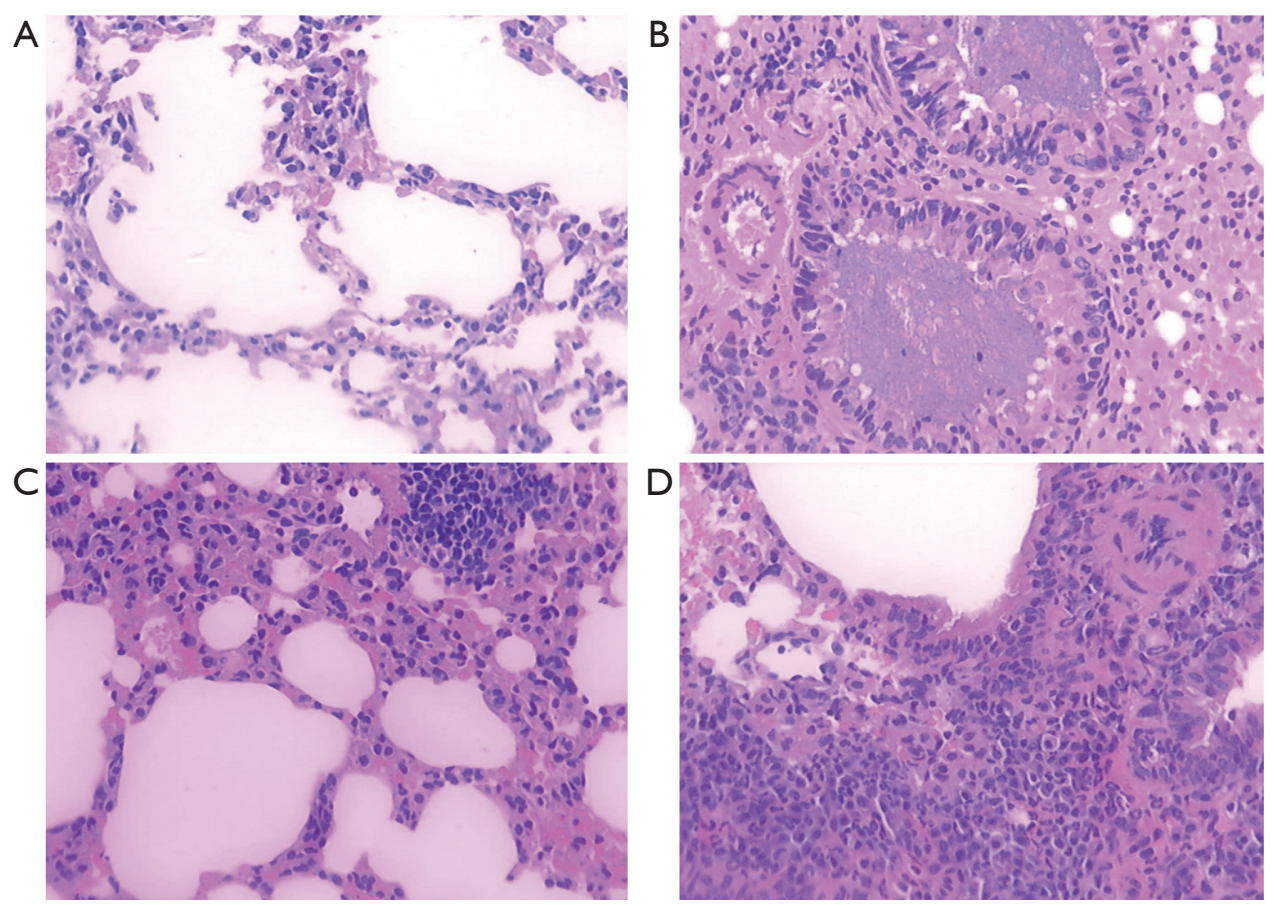

Figure 5 Representative histopathological images of lung tissue (HE staining) were showed: (A) normal group; (B) OVA group; (C) NU7441 group and (D) OVA + vehicle group with magnification of 400x. There were obvious features of airway inflammation in (B) and (D), including inflammatory cell infiltration in the vicinity of bronchioles, alveolar cavity, alveolar septum and surrounding vessels, irregular enlarging of the residual alveolar cavity, and mucus impacted in alveolar cavity. NU7441 attenuate the inflammatory cell infiltration and cilia damage significantly as seen in (C). OVA, ovalbumin.
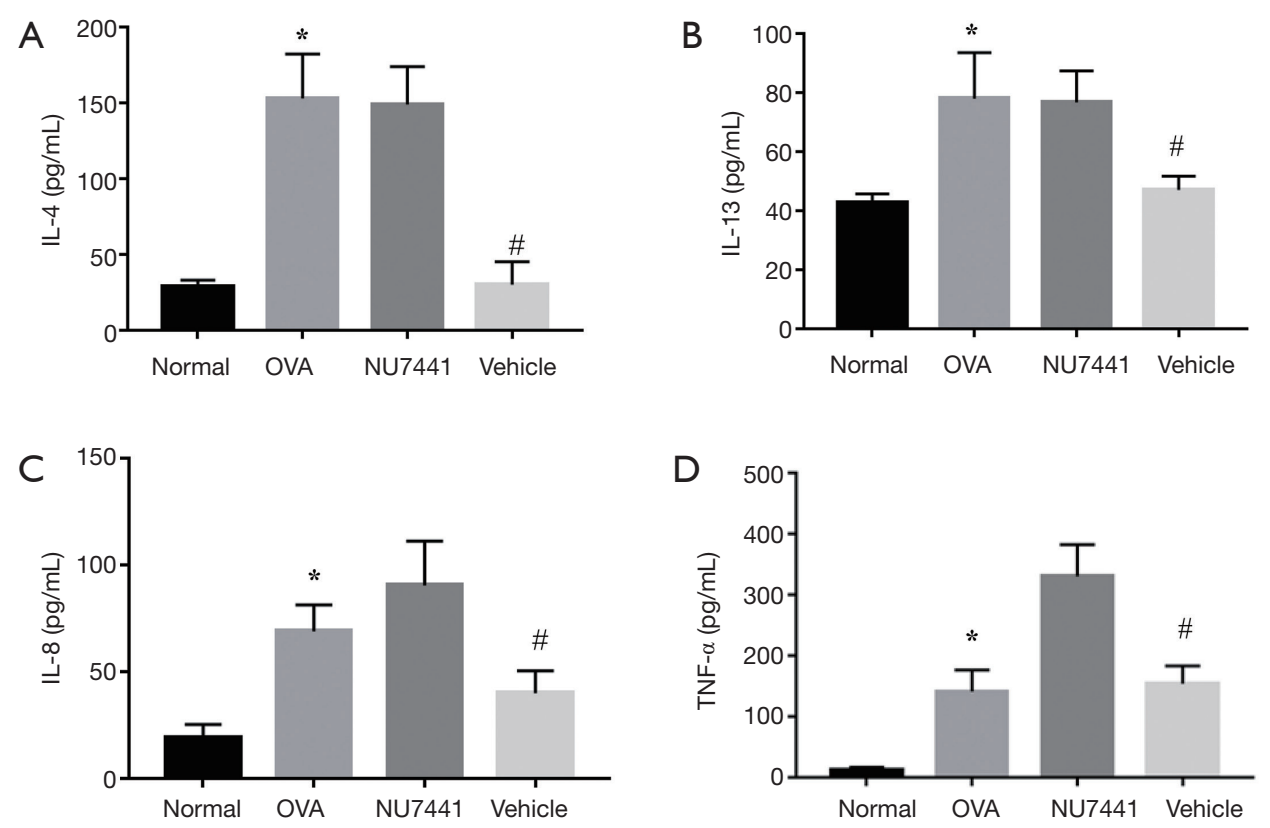

Figure 6 Cytokines (IL-4, IL-13, IL-8, and TNF- $\alpha$ ) in BALF were measured using ELISA (n=9 per group). *, OVA group $v s$. Normal group, $\mathrm{P}<0.001$; \#, NU7441 group vs. OVA + vehicle group, $\mathrm{P}<0.001$. OVA, ovalbumin; BALF, bronchoalveolar lavage fluid; ELISA, enzyme linked immunosorbent assay. 


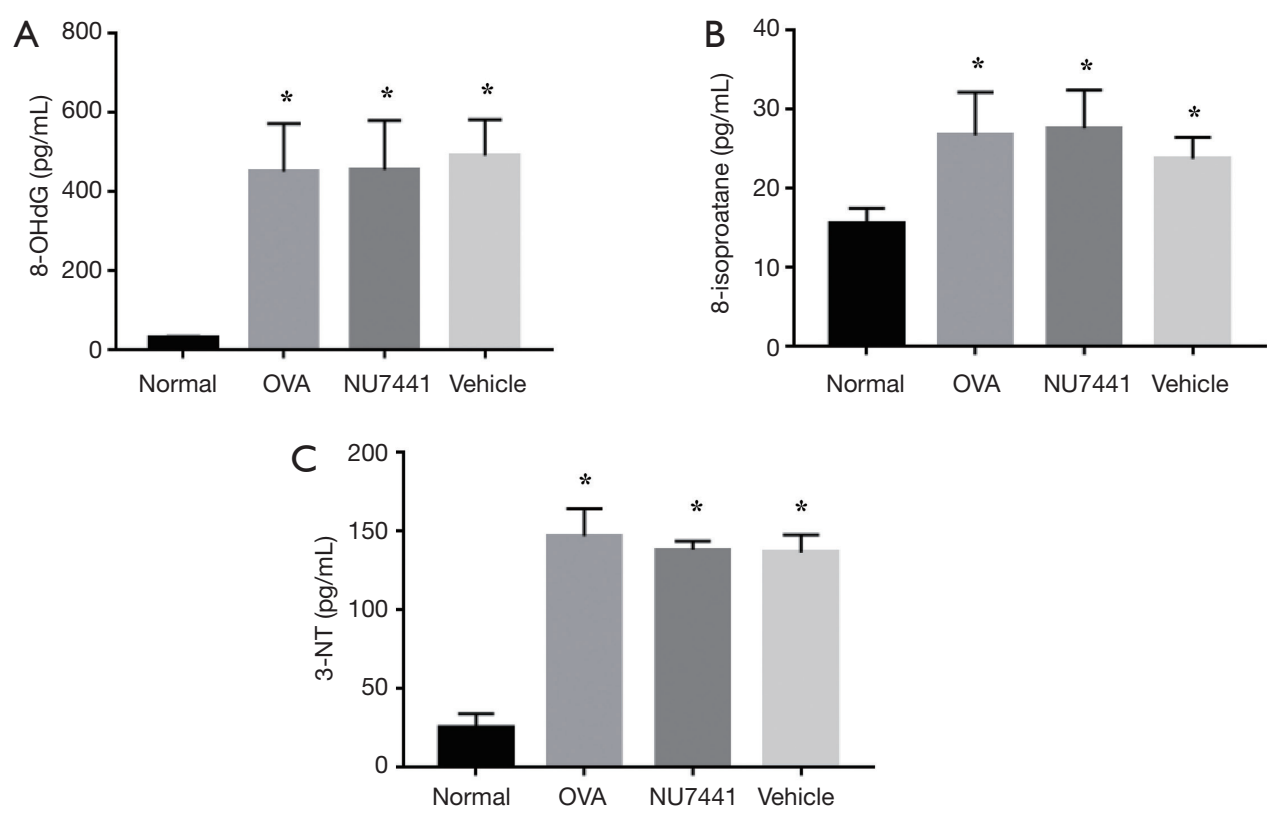

Figure 7 8-isoprostane and 8-OHdG in BALF and 3-NT in lung tissue were measured with ELISA. *, OVA group, NU7441 group, OVA + vehicle group vs. Normal group, $\mathrm{P}<0.05$. OVA, ovalbumin; BALF, bronchoalveolar lavage fluid; ELISA, enzyme linked immunosorbent assay.

\section{DNA damage in BALF cells}

As shown in Figure 8, alkaline comet assay was conducted to measure DNA damage. The alkaline comet assay is a singlecell gel electrophoresis assay for detecting extensive DNA damage including DSB, SSB and base lesions in cells. DNA damage in OVA group mice was potentiated compared with Normal group $(\mathrm{P}<0.05)$. NU7441 enhanced this DNA damage compared with OVA + vehicle group $(\mathrm{P}<0.05)$.

\section{DNA DSBs in lung tissues}

To further detect DNA DSB damage in each group, we choose $\gamma \mathrm{H} 2 \mathrm{AX}$ as a marker. DSBs can be measured visually by immunohistochemistry assay. As shown in Figure 9, in contrast with Normal group, the frequency of $\gamma \mathrm{H} 2 \mathrm{AX}$ positive cells in OVA group was significantly increased $(16.61 \% \pm 2.91 \%$ vs. $0.05 \% \pm 0.13 \%, \mathrm{P}<0.001)$. Vehicle had no effect on it, while NU7441 group augmented frequency of $\gamma \mathrm{H} 2 \mathrm{AX}$-positive cells $(17.34 \% \pm 3.73 \%$ vs. $29.07 \% \pm 5.13 \%$, $\mathrm{P}<0.001)$.

\section{DNA repair proteins in lung tissues}

Four key DNA repair proteins were selected to explore DNA repair responses in asthma: $\gamma \mathrm{H} 2 \mathrm{AX}, \mathrm{Ku} 70, \mathrm{Rad} 51$ and
PARP-1. Ku70 and Rad51 are key proteins in NHEJ and HR repair pathways respectively, and PARP-1 is involved in SSBs repair responses. As shown in Figure 10, levels of 4 proteins were robust augmented in OVA group compared with Normal group $(\mathrm{P}<0.001)$, suggesting that asthma significantly increased the expression of key DNA repair proteins. Compared with OVA + vehicle group, NU7441 markedly increased $\gamma \mathrm{H} 2 \mathrm{AX}$ and Rad51 levels, while $\mathrm{Ku} 70$ and PARP-1 levels were reduced $(\mathrm{P}<0.05)$.

\section{Discussion}

Asthma is a chronic airway inflammatory disease with obvious heterogeneity. Injury and shedding of bronchial epithelial cells is one of the common pathological signs in asthmatic patients, which is considered as a result of chronic airway inflammation in previous view. However, some recent studies have found that the inflammation and oxidative damage that allergen induced are likely to have genotoxic effects on bronchial epithelial cells $(3,15)$. Furthermore, aeroallergens might also have a direct DNAdamaging effect on bronchial epithelium (16). Infiltration of inflammatory cells, especially eosinophils and neutrophils, is one of the important pathological features of asthma. In this study, the number of total cells, eosinophils and neutrophils in asthmatic mice was higher, which evidenced 


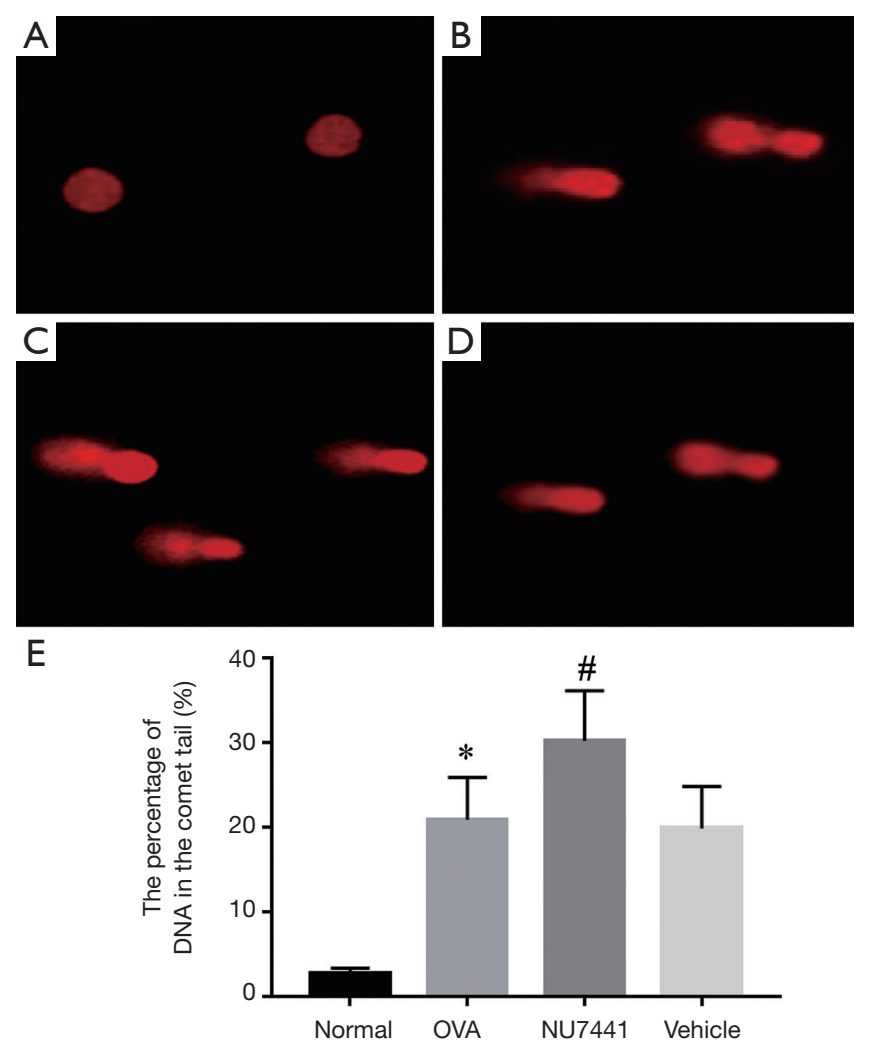

Figure 8 DNA damage was analyzed with alkaline comet assay: (A) Normal group; (B) OVA group; (C) NU7441 group and (D) OVA + vehicle group. *, OVA group vs. Normal group, $\mathrm{P}<0.05$; \#, NU7441 group vs. Vehicle group, $\mathrm{P}<0.05$. OVA, ovalbumin.

that the murine asthmatic model which we established was successful. The major causes of oxidative damage that allergen induced are RONS, which are mainly produced by eosinophils and neutrophils. Moreover, MPO and $\mathrm{EPO}$, the most abundant lysosomal enzyme in neutrophils and eosinophils respectively, can catalyze the formation of hypochlorous acid and hypobromous acid (16). These hypohalous acids can react with superoxide to form oxyradical, which is a highly cytotoxic oxygen free radical (16). Furthermore, there was a study revealed the correlation between these oxidative damage and DNA damage in asthma patients (17). Therefore, it is responsible that oxidative damage and DNA damage play crucial role in manifestation of asthma pathogenesis.

In order to explore the oxidative damage in asthmatic mice, we chose 8 -isoprostane, 8 -OHdG and 3 -NT as indicators to measure oxidative damage (18). 8-isoprostane is a stable terminal product formed by lipid peroxidation
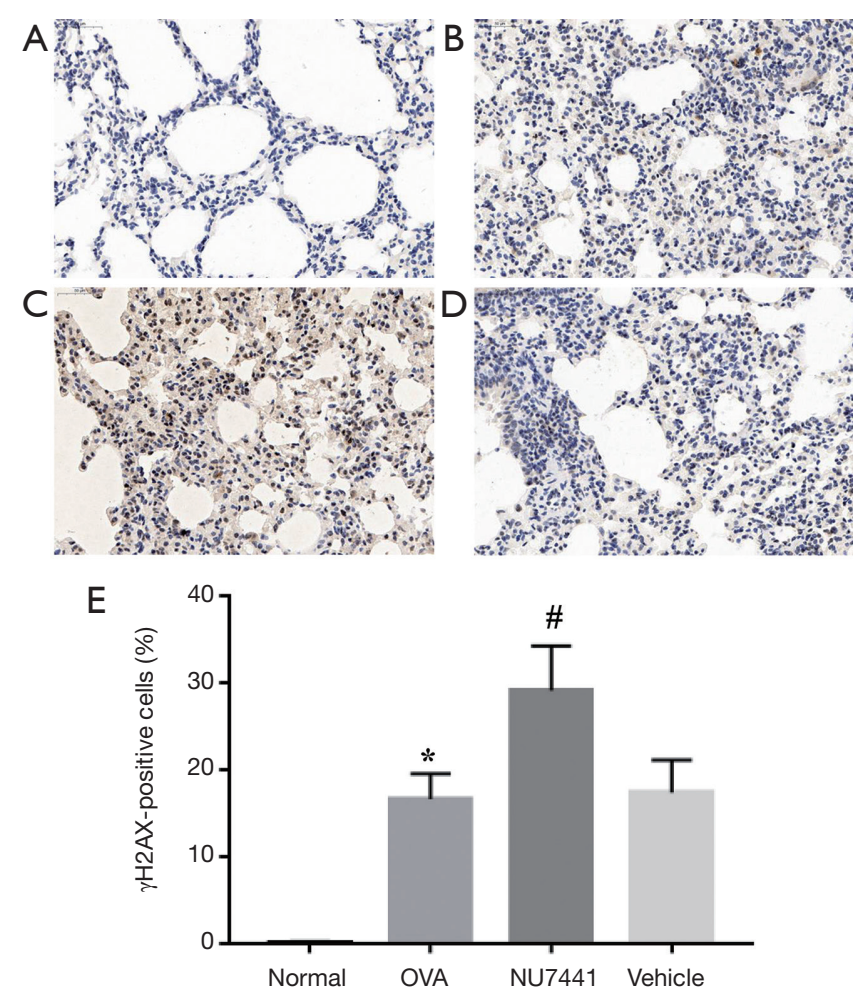

Figure 9 Presented a dramatic difference of immunohistochemistry assay in 4 groups. (A) Normal group; (B) OVA group; (C) NU7441 group; (D) OVA + vehicle group and (E) the percentage of $\gamma \mathrm{H} 2 \mathrm{AX}$-positive cells with magnification of $40 \times$. The frequency of $\gamma \mathrm{H} 2 \mathrm{AX}$ positive cells in (B) was significantly increased compared with (A). (D) had no effect on it, while (C) augmented frequency of $\gamma \mathrm{H} 2 \mathrm{AX}$-positive cells. *, OVA group vs. Normal group, $\mathrm{P}<0.001$; \#, NU7441 group vs. Vehicle group, $\mathrm{P}<0.001$. OVA, ovalbumin.

on cell membrane (18). Because it has a strong polarity, the increase of it can influence the integrity and fluidity of cell membrane. The increase of 8 -isoprostane can lead to the damage to the structure and function of the cells, and cell death eventually (19). 3-NT is formed by oxidation of nitrite by MPO or EPO, both of which are abundant in the asthma. 3-NT also participates in protein denaturation and DNA damage response, which can cause cell death or apoptosis (20). 8-OHdG is a specific product form by guanine oxidative damage in DNA, and the level of it can quantify the oxidative damage of nucleic acid. In this study, we found that OVA challenge resulted in a robust increase in these three indicators, suggesting the oxidative damage to lipids, proteins and nucleic acid were higher in asthmatic mice. 
A
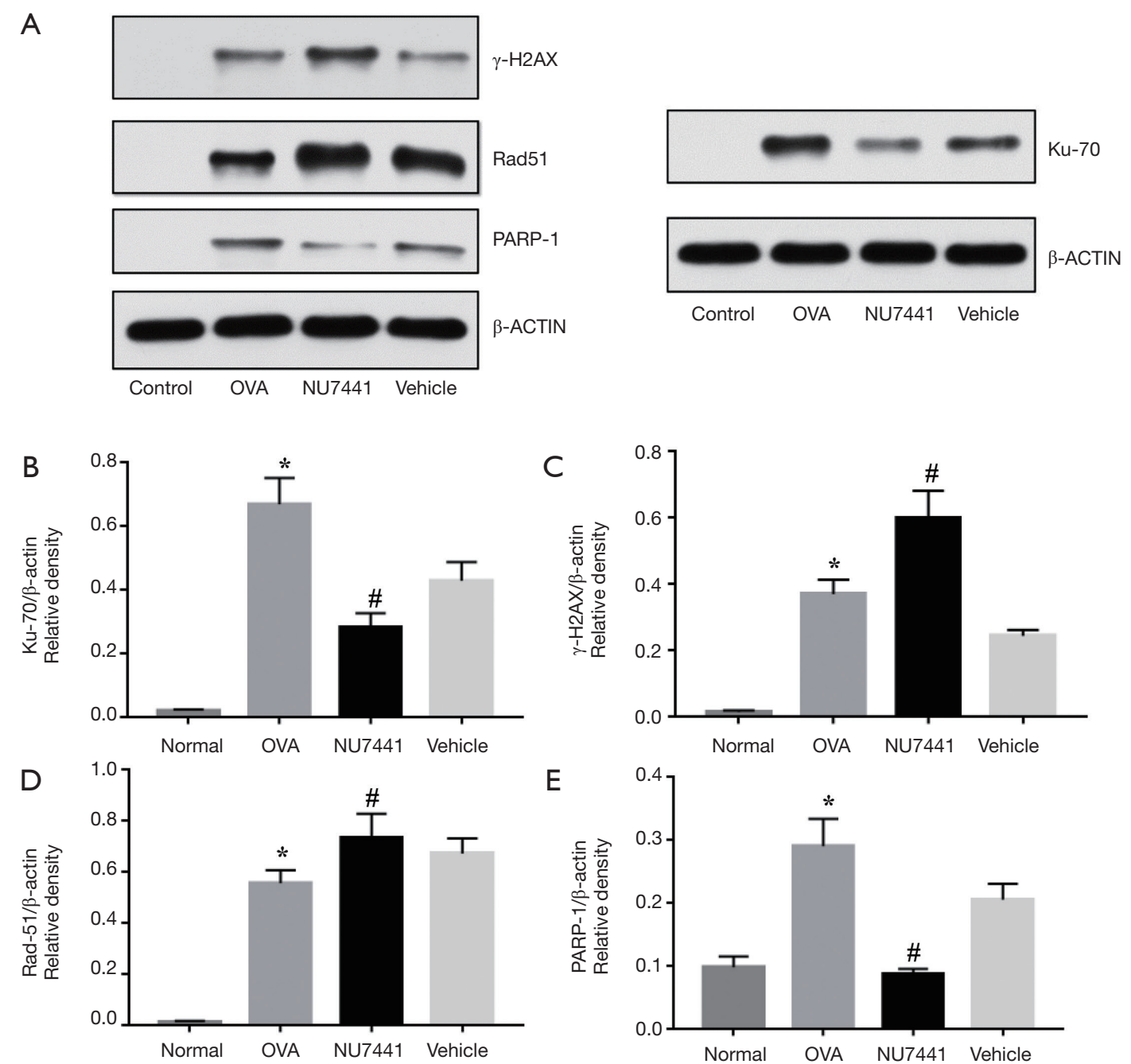

Figure 10 Asthma induces DNA damage responses, as indicated by immunoblotting results of 4 key DNA repair proteins. NU7441 markedly increased $\gamma \mathrm{H} 2 \mathrm{AX}$ and Rad51 levels, while Ku70 and PARP-1 levels were reduced. Immunoblotting images for $\gamma \mathrm{H} 2 \mathrm{AX}, \mathrm{Ku} 70$, Rad51 and PARP1 were shown, $\beta$-actin was used as an internal control (A). Relative density analysis of Ku70 (B), $\gamma \mathrm{H} 2 \mathrm{AX}$ (C), Rad51 (D) and PARP-1 (E) were shown respectively. Bars indicate the mean density ratio \pm SEM (n=4). *, OVA group vs. Normal group, $\mathrm{P}<0.001$; \#, NU7441 group $v s$. OVA + vehicle group, $\mathrm{P}<0.001$. OVA, ovalbumin.

Most DNA damage induced by RONS are base lesions and SSB. Moreover, SSBs can be turned into DSBs if the two SSB is close enough (6). DSB is one of the most toxic damage to cells, and if not repaired in time, it will cause genome rearrangement and even cell death. In order to further explore the DNA DSBs in the lung tissue of asthmatic mice, we selected $\gamma \mathrm{H} 2 \mathrm{AX}$ as a marker by immunohistochemistry and immunoblotting. $\gamma \mathrm{H} 2 \mathrm{AX}$ is one of H2AX chromosome histone family members. When DNA DSBs occurs, the H2AX will be phosphorylated and turned into $\gamma \mathrm{H} 2 \mathrm{AX}$, which plays a key role in DNA damage repairing (21). Moreover, previous study had shown that $\gamma \mathrm{H} 2 \mathrm{AX}$ also participated in monitoring DNA replication processes, which were identifying the stagnation of DNA replication fork and preventing from more severe DNA damage specifically (22). Therefore, $\gamma \mathrm{H} 2 \mathrm{AX}$ is often used as a marker to identify the damage and repair of DNA DSBs. We found that the rate of $\gamma \mathrm{H} 2 \mathrm{AX}$ positive cells in lung tissue of asthmatic mice was significantly higher than that of normal mice, especially in airway epithelium. The result of $\gamma \mathrm{H} 2 \mathrm{AX}$ levels by Western immunoblotting also showed a significant increase in asthmatic mice. We also conducted 
a comet assay on BALF cells, and the results showed that DNA damage was more severe in asthmatic mice. Thus, there was a significant increase in DNA damage in OVAinduced asthma by both qualitative and quantitative analysis.

It should be noted that DSBs can not only be formed pathologically, but also be formed endogenously in immature $\mathrm{B}$ and $\mathrm{T}$ lymphocytes during $\mathrm{V}(\mathrm{D}) \mathrm{J}$ recombination physiologically (23). V(D)J recombination is the necessary process of adaptive immune response, which occurs only in lymphoid tissues. Only mature B and T lymphocytes that after $\mathrm{V}(\mathrm{D}) \mathrm{J}$ recombination can migrate to the lungs and other tissues (24). In this study, in order to avoid the confusion caused by V(D)J recombination, we carefully cleaned up the lymph nodes and fat tissues and when collecting samples. Therefore, the DSBs detected in tour study was unlikely to be from $\mathrm{V}(\mathrm{D}) \mathrm{J}$ recombination.

To learn about the repair process of DNA damage in asthmatic mice, we also detected 3 kinds of DNA damage repair proteins: $\operatorname{Rad} 51, \mathrm{Ku} 70$ and PARP-1. Ku70 and Rad51 are key proteins in NHEJ and HR repair pathways respectively, while PARP-1 plays an important role in DNA SSBs. There were studies confirmed that the level of Ku70 in asthmatic lung tissue was significantly higher than that in healthy lung tissue, suggesting that the up regulation of DNA damage repair protein in asthma patients might be important in the pathogenesis of asthma (25). In this study, we found significantly increased levels of these 3 DNA damage repair proteins in OVA-challenged mice, suggesting that DNA repair response was augmented in the asthmatic mice.

To further explore DNA damage repair condition in $t$ asthma, we chose DNA-PKcs inhibitor NU7441, which participates NHEJ pathway, to study it. NU7441 is a novel small molecule inhibitor of DNA-PKcs. Compared with the previous inhibitor, NU7441 has the characteristics of low toxicity, good targeting and high stability in vivo (11). After administration of NU7441, compared with the ordinary asthma mice, we had not find significant changes in AHR indicators (RI, RE and Cydn), and the levels of oxidative damage indicators (8-isoprostane, 3-NT and 8-OhdG), suggesting that inhibition of DNA-PKcs did not aggravate the airway hyperresponsiveness and oxidative damage in asthmatic mice. AHR and oxidative damage in asthma are influenced by multiple factors, and DNA damage might be only one of these factors. In addition, the duration of NU7441 intervention in this study may not be sufficient to observe a significant difference in AHR and oxidative damage.
Our study also showed effects of NU7441 on DNA damage and repair in asthmatic mice. The comet assay and $\gamma \mathrm{H} 2 \mathrm{AX}$ detection by immunohistochemistry and immunoblotting revealed that NU7441 augmented DNA damage in allergic airways significantly. Although some researches had found that inhibition of DNA-PKcs can suppress phosphorylation of H2AX (26), NU7441 in our study increased $\gamma \mathrm{H} 2 \mathrm{AX}$ levels significantly. One reasonable explanation was that although NU7441 inhibited the ability of DNA-PKes to form $\gamma \mathrm{H} 2 \mathrm{AX}$, high DNA DSBs up-regulated the expression of some other enzymes which can also activate H2AX [such as expression of PI3 kinaseassociated protein kinase (PK)]. Thus, NU7441 led a strong $\gamma \mathrm{H} 2 \mathrm{AX}$ signal in asthmatic mice. In addition, NU7441 administration also up-regulated the expression of Rad-1, suggesting that when the NHEJ pathway is suppressed, the RAD51-associated HR repair pathway is feedback enhanced (27). There was a significant decrease in the express of $\mathrm{Ku} 70$. One possible explanation is that NU7441 breaks the stable complex of DNA-PKcs and Ku70, potentially leading to Ku70 degradation (28).

Interestingly, we found NU7441 had beneficial effects on asthma. The result of total and differential cell counts and cytokines in the BALF showed that NU7441 can repress airway inflammation significantly. There were evidences that only less than $1 \%$ of DNA-PK in human body is needed to repair DNA damage $(7,29)$, suggesting that DNA-PK not only mediates DSB repair by NHEJ, but also regulates innate immune responses and pro-inflammatory signaling pathways (30). The previous studies found that NU7441 can reduce Th2-related airway inflammation and AHR effectively in asthmatic mice by Akt and NF- $\mathrm{KB}$ signaling pathway $(8,9)$. There were also researchers found that DNA-PK affected cell drug resistance through PI3KAkt pathway and NU7441 can restore drug sensitivity (10). These findings reveal that DNA-PK has a novel and unexpected function on inflammation of asthma and might have an impact on the drug-resistance.

In summary, we found that high levels of oxidative damage, DNA damage and DNA repair protein expression in OVA-induced asthmatic mice. DNA-PKcs inhibitor NU7441 can influence the DNA DSBs and DNA damage repair protein expression, suggesting the potential effects of DNA damage and repair in the pathogenesis of asthma. In addition, we initially found that NU7441 had an antiinflammation effect on asthmatic airway, suggesting that DNA-PK might have other functions besides DNA damage repair enzyme, which needs further research to explore. 


\section{Acknowledgements}

We would like to thank Q Huang, Q Wei and Q Luo, whose names are not included in the list of authors but nonetheless contributed to the work of this team.

\section{Footnote}

Conflicts of Interest: The authors have no conflicts of interest to declare.

Ethical Statement: This study was approved by the Animal Ethics Committee of China-Japan Friendship Hospital (ID of the ethic approval: 170105).

\section{References}

1. Masoli M, Fabian D, Holt S, et al. The global burden of asthma: executive summary of the GINA Dissemination Committee report. Allergy 2004;59:469-78.

2. Lambrecht BN, Hammad H. The immunology of asthma. Nat Immunol 2015;16:45-56.

3. Ho WE, Cheng C, Peh HY, et al. Anti-malarial drug artesunate ameliorates oxidative lung damage in experimental allergic asthma. Free Radic Biol Med 2012;53:498-507.

4. Kiziltepe T, Yan A, Dong M, et al. Delineation of the chemical pathways underlying nitric oxide-induced homologous recombination in mammalian cells. Chem Biol 2005;12:357-69.

5. Mutamba JT, Svilar D, Prasongtanakij S, et al. XRCC1 and base excision repair balance in response to nitric oxide. DNA Repair (Amst) 2011;10:1282-93.

6. Panier S, Durocher D. Push back to respond better: regulatory inhibition of the DNA double-strand break response. Nat Rev Mol Cell Biol 2013;14:661-72.

7. Woodbine L, Neal JA, Sasi NK, et al. PRKDC mutations in a SCID patient with profound neurological abnormalities. J Clin Invest 2013;123:2969-80.

8. Ghonim MA, Pyakurel K, Ju J, et al. DNA-dependent protein kinase inhibition blocks asthma in mice and modulates human endothelial and $\mathrm{CD}^{+}{ }^{+} \mathrm{T}$-cell function without causing severe combined immunodeficiency. J Allergy Clin Immunol 2015;135:425-40.

9. Mishra A, Brown AL, Yao X, et al. Dendritic cells induce Th2-mediated airway inflammatory responses to house dust mite via DNA-dependent protein kinase. Nat Commun 2015;6:6224.
10. Xi G, Hayes E, Lewis R, et al. CD133 and DNA-PK regulate MDR1 via the PI3K- or Akt-NF- $\mathrm{KB}$ pathway in multidrug-resistant glioblastoma cells in vitro. Oncogene 2016;35:241-50. Retraction in: Oncogene 2016;35:5576.

11. Zhao Y, Thomas HD, Batey MA, et al. Preclinical evaluation of a potent novel DNA-dependent protein kinase inhibitor NU7441. Cancer Res 2006;66:5354-62.

12. Hao M, Lin J, Shu J, et al. Clarithromycin might attenuate the airway inflammation of smoke-exposed asthmatic mice via affecting HDAC2. J Thorac Dis 2015;7:1189-97.

13. Luo Q, Lin J, Zhang L, et al. The anti-malaria drug artesunate inhibits cigarette smoke and ovalbumin concurrent exposure-induced airway inflammation and might reverse glucocorticoid insensitivity. Int Immunopharmacol 2015;29:235-45.

14. Hininger I, Chollat-Namy A, Sauvaigo S, et al. Assessment of DNA damage by comet assay on frozen total blood: method and evaluation in smokers and non-smokers. Mutat Res 2004;558:75-80.

15. Sugiura H, Ichinose $M$. Nitrative stress in inflammatory lung diseases. Nitric Oxide 2011;25:138-44.

16. Comhair SA, Erzurum SC. Redox control of asthma: molecular mechanisms and therapeutic opportunities. Antioxid Redox Signal 2010;12:93-124.

17. Zeyrek D, Cakmak A, Atas A, et al. DNA damage in children with asthma bronchiale and its association with oxidative and antioxidative measurements. Pediatr Allergy Immunol 2009;20:370-6.

18. Montuschi P, Barnes PJ, Roberts LJ 2nd. Isoprostanes: markers and mediators of oxidative stress. FASEB J 2004;18:1791-800.

19. Schwedhelm E, Tsikas D, Durand T, et al. Tandem mass spectrometric quantification of 8-iso-prostaglandin F2alpha and its metabolite 2,3-dinor-5,6-dihydro-8-isoprostaglandin F2alpha in human urine. J Chromatogr B Biomed Sci Appl 2000;744:99-112.

20. Brennan ML, Wu W, Fu X, et al. A tale of two controversies: defining both the role of peroxidases in nitrotyrosine formation in vivo using eosinophil peroxidase and myeloperoxidase-deficient mice, and the nature of peroxidase-generated reactive nitrogen species. J Biol Chem 2002;277:17415-27.

21. Li M, Lu LY, Yang CY, et al. The FHA and BRCT domains recognize ADP-ribosylation during DNA damage response. Genes Dev 2013;27:1752-68.

22. Ward IM, Chen J. Histone H2AX is phosphorylated in an ATR-dependent manner in response to replicational stress. J Biol Chem 2001;276:47759-62. 
23. Chen HT, Bhandoola A, Difilippantonio MJ, et al. Response to RAG-mediated VDJ cleavage by NBS1 and gamma-H2AX. Science 2000;290:1962-5.

24. Parkin J, Cohen B. An overview of the immune system. Lancet 2001;357:1777-89.

25. Chan TK, Loh XY, Peh HY, et al. House dust miteinduced asthma causes oxidative damage and DNA double-strand breaks in the lungs. J Allergy Clin Immunol 2016;138:84-96.e1.

26. Jackson SP, Bartek J. The DNA-damage response in human biology and disease. Nature 2009;461:1071-8.

Cite this article as: Wang Y, Lin J, Shu J, Li H, Ren Z. Oxidative damage and DNA damage in lungs of an ovalbumin-induced asthmatic murine model. J Thorac Dis 2018;10(8):4819-4830. doi: 10.21037/jtd.2018.07.74
27. Shrivastav M, Miller CA, De Haro LP, et al. DNA-PKcs and ATM co-regulate DNA double-strand break repair. DNA Repair (Amst) 2009;8:920-9.

28. Davis AJ, Chen BP, Chen DJ. DNA-PK: a dynamic enzyme in a versatile DSB repair pathway. DNA Repair (Amst) 2014;17:21-9.

29. Meek K, Dang V, Lees-Miller SP. DNA-PK: the means to justify the ends? Adv Immunol 2008;99:33-58.

30. Kong X, Shen Y, Jiang N, et al. Emerging roles of DNAPK besides DNA repair. Cell Signal 2011;23:1273-80. 